\title{
Teaching non-technical skills: the patient centered approach
}

\author{
Gianluca Casali ${ }^{1}$, Gareth Lock $^{2}$, Nuria M. Novoa ${ }^{3}$ \\ ${ }^{1}$ Thoracic Surgery, University Hospital Bristol NHS Foundation Trust, Bristol, UK; ${ }^{2}$ Human in the System Consulting Limited, Malmesbury, UK; \\ ${ }^{3}$ Service of Thoracic Surgery, University Hospital of Salamanca, Salamanca, Spain \\ Contributions: (I) Conception and design: All authors; (II) Administrative support: None; (III) Provision of study materials or patients: None; (IV) \\ Collection and assembly of data: None; (V) Data analysis and interpretation: None; (VI) Manuscript writing: All authors; (VII) Final approval of \\ manuscript: All authors. \\ Correspondence to: Dr. Nuria M. Novoa. Thoracic Surgery Service, Paseo de San Vicente 52-84, 37007 Salamanca, Spain. Email: nuria.novoa@usal.es.
}

\begin{abstract}
The surgical setting is a highly complex environment where, in ideal conditions, everything should be under control to ensure a positive outcome. However, the existing complexity opens the possibility for multiple failures along the process and many of those failures are related to what is call the non-technical skills of the members of the team. We cannot eradicate human error, but we can try to avoid future mistakes in our daily practice introducing the awareness for providing a high-quality care in which patient safety is crucial. This paper presents an easy approach to concepts and teaching possibilities of those non-technical skills.
\end{abstract}

Keywords: Non-technical skills; safe surgery; safety culture; adverse events; communication; situational awareness

Submitted Oct 02, 2018. Accepted for publication Jan 14, 2019.

doi: $10.21037 /$ jtd.2019.01.48

View this article at: http://dx.doi.org/10.21037/jtd.2019.01.48

\section{Introduction}

The surgical setting is a highly complex environment where in ideal conditions everything should be under control to ensure a positive outcome. However, the existing complexity opens the possibility for multiple failures along the process. It is estimated that, in $1984,41 \%$ of all the hospital errors occurred in the operating room but only $14 \%$ were caused by negligent care (1).

\section{Never events}

In 2013, Mehtsun et al. (2) published a rigorous investigation on US national malpractice claims and found that near 80,000 never-events occurred in the US-hospitals from 1990 to 2010. Never events are adverse events that are serious and should be largely preventable. This sparse data gives a global idea of the magnitude of what patient harm means. No matter if the failures had or had not relevant impact on the patient, they should not happen. Unsafe procedures have very important costs in patient's disability, loss of personal satisfaction, deaths and health care expenses (3).
However, it must be recognized that never events only occur when always needed conditions are absent (4) and therefore the focus should move from just trying to 'fix' the active failures which occur, but to address the 'latent pathogens' (5) which exist in the system.

\section{Safety as an emerging property of a system}

The solution is to provide the knowledge about what to focus on, and more importantly, to encourage a global change in culture. We need to implement a culture whereby the safety of patients and staff is managed as part of the same business model that healthcare operates under, because safety, realistically, can never be the first goal of an organizational all the time if it is to remain commercially viable.

Safety culture in the health care system has been defined as an integrated pattern of individual and group behaviors, based on shared beliefs and values, that continuously seeks to minimize patient harm that may result from the process of delivering care (6). Establishing a safety culture is one of the most effective strategies to achieve sustainable 
improvement in patient safety (7) and yet is one of the most challenging given the huge number of stakeholders within the system. One of the reasons for this reticence to change is that a Safety Culture cannot be forcefully developed, rather it is an emergent property of the organization. Compliance can be forced into an organization, but that isn't the same as safety.

\section{Safety management system (SMS)}

From other high-risk industries such as nuclear, aviation, food management or oil and gas, we have learned that information on safety alone is of little use.

Patient safety needs an organized broad approach which is referred to as SMS (8). The aim of a SMS is to measure safety indicators constantly (8), review them and offer feedback for continual improvement. To achieve this goal there should be in place clear policies and structural elements (dedicated safety officers, governance committees...) that implement a global strategy of safety.

Some of the elements of a SMS have been present in the healthcare community for a long time. Information on human performance and safety is not usually an issue. Some of the structural elements that allow the collection and review of the data are present in most of the surgical departments. Mortality and morbidity meetings are commonplace but whether they identify systemic issues is not clear.

What is still missing is a global focus on safety that is fully informed by the advances in other high-risk domains. Part of the problem is the absence of strong evidence that shows a direct return on investment for human factors training and safety culture programs. The large number of variables involved in the safe performance of a complex system creates a barrier to the possibility of designing and running the kind of study needed to provide this evidence and unfortunately while this discussion progresses more patients will die unnecessarily. Healthcare-based Crew Resource Management (CRM) programs which have delivered a return of investment have required multiple years to deliver their results $(9,10)$. Sometimes it seems that creating change in healthcare is difficult, even when evidence is provided (11).

\section{Patient safety and surgery}

Notwithstanding the above, it is remarkable that in a very short period, the amount of research and knowledge has increased exponentially reflecting the great interest in the field. In 1990, a PubMed search for "patient safety" yielded 27 documents, in 2011 more than 1900 documents (12) and currently, in 2018, more than 128,000 documents. One of the most significant outcomes is that unsafe procedures mean low quality performance (13) and this has led to a high-level of interest focusing on quality improvements.

\section{Checklists}

A simple measure that had shown a great impact improving safety: the introduction in our daily routine of cross check controls based on checklists $(14,15)$. The application of checklists into highly dynamic and complex environments reduces ambiguity and uncertainty by helping create a shared mental, forces a 'slow down' of the team to reduce negative effects of cognitively-biased decisions and improves communications leading to more effective teamwork. This technique, imported from other high-risk industries or activities, summarizes a great effort for readdressing individual routine check controls toward a systematic and consistent attempt of control involving all the essential personnel in the process at the same time (16). However, checklists are not a panacea and require informed design and the application of non-technical skills to be effective (17).

\section{Clinical practice guidelines}

Furthermore, another interesting advance is the development of standardized processes based on evidencebased data (18). The recognition of the importance of agreeing on precise steps for diagnosis and treatment of diseases based on data gathered from the most relevant and specific populations is another tool that has gained traction within our units.

\section{Safety culture}

Training, mentoring and coaching to create a culture whereby high-performance leads to a safe environment for patients and staff is a process that should be considered as critical in all programs dedicated to professionals in healthcare. Doctors, nurses and, even administrative staff of the units, policymakers and regulators all have an impact on patient safety and so should be educated. For instance, concepts such as never-events, near-miss analysis, safety climate, situation awareness, decision making, effective communication skills, teamwork and leadership 
are all essential components of this culture. Safety and the associated high-performance skillsets should not be a 'bolton', but rather be part of the daily routine. For instance, in the surgical settings, especially at the operating room, it would be simplistic to say that the 'human factor' is the leading cause of errors (19) as human performance is complex in nature and failures occur up and down the organizational system and not just the 'sharp end' which is where it is often attributed. Consequently, if we want to improve patient safety, we need to look at the variability of human performance at an individual level, a team level and an organizational level and how these all impact the patient and not just the performance of the surgeon, unit or hospital.

\section{Human error and adverse event analysis}

We cannot eradicate human error. Therefore, the main aim of any adverse event analysis is to improve future error prevention, error trapping and/or error mitigation. This is achieved by creating an understanding of 'the how' and 'the why' the event occurred in the manner it did. It is not achieved by punishing those involved, despite this being a societal and cultural expectation (20). Such a retributive approach reduces organizational and personal learning leading to the same errors and error producing conditions being repeated. Unfortunately, the fear of harsh judgment and humiliation, the possibility of mistrust and hostile behaviors or job loss, among others, makes such data collection and analysis very difficult (21). Specific frameworks such as Human Factors Analysis and Classification System (HFACS) (22) can help improve the scope of investigations by looking at systemic issues in a recognized framework, but care should be taken when categorizing events as those undertaking the analysis will add considerable bias to the process when it comes to contributory factors (23). One way around this is a narrative process, such as Learning Reviews and Appreciative Inquiries, as these have been shown to have a greater impact on operational learning than simplistic categorizations (24).

Recognizing the need to spread this knowledge, agencies have started educational programs to spread this knowledge. The initial idea and development took place at the University of Aberdeen in Scotland funded by the Royal College of Surgeons of Edinburgh [RCS(Ed)] and NHS Education for Scotland. There, the first Non-Technical Skills for Surgeons (NOTSS) course was launched. Their aims, as described by one of the leaders of the initiative, was to develop and test an educational system for assessment and training based on observable behavioral skills in the intraoperative phase of surgery (25).

The aim of this paper is providing an initial and global overview of what and how must be learned to increase our knowledge of these non-technical skills.

\section{Technical skills vs. non-technical skills}

The analysis of accidents and near misses in aviation and high-risk industries revealed that frequently the failure was not linked to a lack of knowledge or experience but rather the failure was instead from a group of 'soft skills' that were initially labelled as "non-technical" to distinguish them from those which were 'technical' i.e. pure 'stick and rudder' piloting skills.

The Chartered Institute for Ergonomics and Human Factors defines non-technical skills as "The cognitive and social skills that contribute to safe and efficient task performance" (26). The RCS (Ed) defines them as "cognitive and interpersonal skills that include: situational awareness, decision making, communication, teamwork and leadership" (27). To help improve surgical team's performance, the RCS (Ed) developed and subsequently endorsed a behavioral marker scheme that allows observers to objectively observe the presence/absence of a skill and facilitate feedback. However, Graham et al. (28) have shown that this is not easy for observers to learn a behavioral markers scheme in a short period of time and provide reliable assessments between raters. One topic which doesn't immediately fit into the 'skills' concept but comes under the banner of human performance is the need to understand the impact of 'performance shaping factors' on both technical and non-technical skills.

The overall performance of teams and individuals correlate with the level of technical skills and non-technical skills. Salas and coworkers (29) described this as linking taskwork (often associated with individual technical skills) with teamwork (combining technical and non-technical skills within the boundaries of organizational goals and limitations). In the surgical context, the ability, for example, to anastomose correctly blood vessels is a technical skill. This skill can be practiced in isolation in a training facility and a surgeon can become proficient at it. When the technical performance is moved into an operating theatre, the success of the procedure hinges on the ability to perform correctly the anastomosis (technical skill) but depends also heavily on situation awareness (gathering 
of information, understanding of information, ability to project and anticipate future states), decision making (considering options, selecting and communicating options, implementing and reviewing decisions), communication and teamwork (exchanging information, establishing a common understanding, coordinating team activities), leadership (setting and maintaining standards, supporting others, coping with pressure) and at the same time being conscious of fatigue, time pressures and other issues caused by internal and external stressors.

However, while we recognise that technical skills are not enough, developing non-technical skills in isolation of others e.g., leadership, is not enough either. Both technical and nontechnical are interdependent. As technical skill competence improves, then more mental capacity is available to deal with other skills such as situational awareness, decision-making, teamwork and leadership. Furthermore, without effective teamwork and communications, situational awareness within and across the teams cannot be improved and without improved situational awareness, improved decision-making is not possible. Finally, without undertaking candid and open post-event debriefs, analyses and learning reviews, the risk of patient harm will remain (or increase) because the same errors will be repeated.

\section{What to teach}

\section{Situational awareness}

Endsley (30) defined situational awareness as "the perception of the elements in the environment within a volume of time and space, the comprehension of their meaning, and the projection of their status in the near future." In simple terms, Situational Awareness is being aware of what is happening around you now, what it means to you now and what is likely to happen in the near future, all based on previous experiences and knowledge.

Our brain has evolved to be able to pay attention to a portion of the events that are constantly happening around us, events which we perceive to be important and relevant at that time. When we focus, our awareness is like a spotlight. It shines bright and highlights a small area of the surrounding area. For example, during a complex procedure, out of the many events and issues that are constantly arising, we can pay attention and focus only on the task at hand and ignore the apparently irrelevant. Unfortunately, that same power also has a dark side because activities continue to happen outside the spotlight and these can be missed, potentially leading to vital pieces of information being omitted from the decision-making process.

'Loss of situational awareness' is a term normally used to describe this phenomenon and often appears in adverse event investigation reports. However, due to the limited bandwidth of our perception and information processing systems, the 'awareness' we have is not 'lost' it is just pointing in the direction of something which is perceived to be more relevant or important, an omission which is obvious in hindsight. Wickens (31) describes this cognitive tunneling as the "allocation of attention to a particular channel of information, diagnostic hypothesis or task goal, for a duration that is longer than optimal, given the expected cost of neglecting events on other channels, failing to consider other hypotheses, or failing to perform other tasks." For example, in 1996 an Eastern Airlines airliner crashed in the Florida Everglades at night killing all onboard as the pilots and flight engineer were focused on a light-bulb associated with the landing gear configuration while the aircraft was in a imperceptible shallow dive. Unfortunately, this same cognitive limitation led to irreversible brain damage to a patient when the two anesthetists who were attempting to get an airway lost awareness of the time (32).

\section{Improving situational awareness}

While situational awareness can be improved, first there needs to be an understanding of how the perceptive capability of the senses and brain works. Research from Hyman (33) has demonstrated the ability of shared awareness to be greater than the individual awareness in a cognitively-challenging scenario. However, for this to work, effective communication, leadership and teamwork is needed. The challenge is that as an individual working in a complex environment we usually become aware of our cognitive tunneling when we regain situational awareness. Strategies to improve situational awareness include honest, open and candid debriefs and delegation of 'oversight tasks' to others within the operating theatre when workload is expected to be high using a positive handover in a similar manner to pilots handing over control of the aircraft "You have control", "I have control", "You have control" and returned when suitable.

\section{Decision making}

Situational awareness and decision-making might be thought of as discrete activities but, in reality, there is an 
overlap. Decision making sits at the heart of achieving good outcomes. It can be defined as the process of reaching a judgement or choosing an option to meet the needs of a given situation (34). It requires an individual or team to create or have a mental model of the situation, generate or determine one or more options which relate to that model, decide on a course of action and then review the outcome. This process keeps repeating until the original or emerging goals have been achieved.

Some different decision-making models have been presented in the research which follow two key processes:

(I) Sub-conscious perception, processing and 'choice';

(II) Logical and discrete steps where the problem, options and decisions are made in slow-time.

Ideally, we would like all the elements relevant for the decision to be made available ahead of time, for them to be known with a high level of certainty, to have enough time to assess all the information which allows the formulation of numerous options and then choose the best among them using some criterion. However, in healthcare, professionals are frequently in situations where not all the relevant elements are known, the time available is short and therefore recognizing relevant and important elements using a highly-developed level of situational awareness is key.

Decisions are based on mental models, some have real clarity, some are incomplete and require interpretation and updates based on current data and information. Healthcare professionals can improve those models over time through training and practicing the identification of antecedents, signs and symptoms, creating probable solutions and communicating them to the team. The real-life feedback (successes and failures), combined with coaching and mentoring, helps refine the mental models that gradually, with time, tend to become more and more complex because experience and seniority increases the likelihood of having to deal with more difficult cases.

As an example, the mental model of a bleeding in theatre differs significantly between a trainee and an expert surgeon. The mental model of a junior trainee would include the ability to recognize a major bleeding (awareness) and most likely the automatic response of apply pressure and call for help. The mental model of an expert surgeon would include the ability to appreciate several nuances about the source of bleeding, and the appropriate response apply pressure, use of hemostatic products, need for proximal clamping, suturing...) based on the mechanism of injury (avulsion of artery, proximal laceration, distal laceration, division...) The expert surgeon's mental model will also include, depending on the severity of the bleeding, a number of additional actions like: activating blood bank, gathering additional equipment. Klein et al. (35) showed that experts were able to identify key elements more quickly and subsequently made better, faster decisions. Novices often waited until they had a surplus of elements before deciding, and in some cases, this was too late.

Flin et al. (34) describe 4 principal methods for decisionmaking:

(I) Intuitive (recognition-primed);

(II) Rule based;

(III) Choice among different options;

(IV) Creative.

Each of these methods focuses on different ways to perceive, decode and determine possible solutions for the current situation. However, what they have in common is that they all require a level of previous experience, including failures, for the models to be useful.

\section{Improving decision-making}

Determining what the best method of decision-making can be difficult. One way to improve this is to develop an understanding and practice of the Cynefin framework (36). The framework looks at four dimensions; simple, complicated, complex and chaotic and the way in which information is derived and then decisions are made based on the element in which the problem sits (Figure 1). However, van Beurden et al. (38) caution against using this as a simple $2 \times 2$ matrix in a reductionist manner, rather it should be used in a way of understanding the situation to develop information gathering and decision-making strategies, especially as problems move from one element to another as time progresses.

One of the continual challenges faced by the medical profession is that there is a difference between practicing decision-making in a classroom/exam setting and real-life decision-making which takes place in the clinical setting. As expected, the performance of individuals and teams is likely to be significantly different in the two contexts. For a long-time, the missing link has been the ability to develop in an environment which allow to learn from failures without causing patient harm. Aviation has managed this conundrum for some time using simulators which range from procedural trainers through to high-fidelity mission simulators. The healthcare world is catching up and during the last decade or so, the use of simulators in healthcare simulation centers has increased. This has facilitated the 


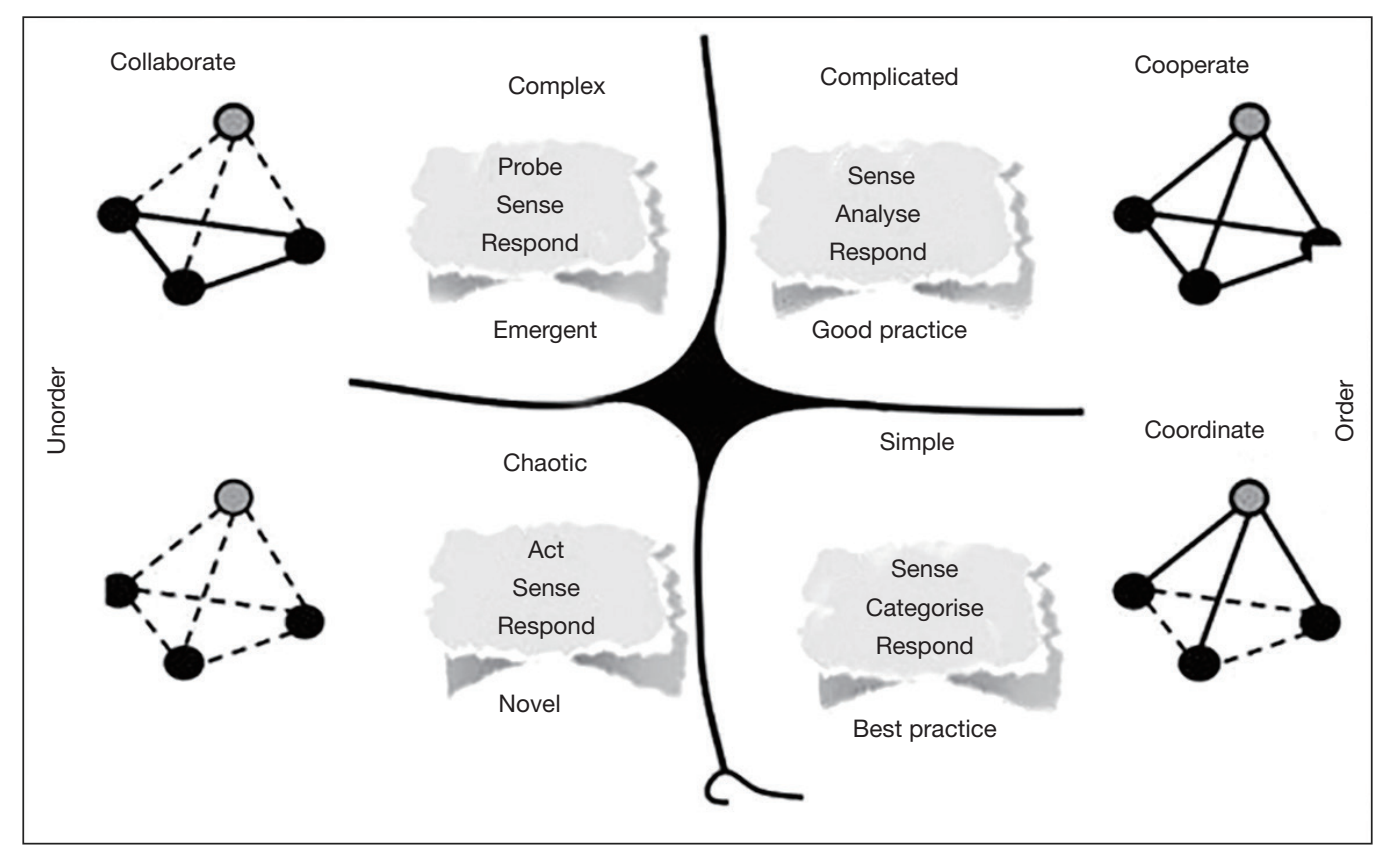

Figure 1 The Cynefin Framework, adapted from Snowden (37). Tetrahedrons show the most appropriate management model. (Apex circle, manager. Base circles, other staff/stakeholders. Solid line, strong connection. Dashed line, weak connection). The central domain of 'disorder' is shown in black (38).

joint training of teams around clinical scenarios and the simultaneous development of individuals and teams with regards to different aspects of technical and non-technical skills. However, the real power of simulation isn't in the task execution, but in the debrief, a process which needs to look at both technical and non-technical skills.

\section{Communication}

Communication is the exchange of information among different members of a team or among different areas of a business. It includes technical information but also the sharing of feelings and emotions. Effective communication is not just exchanging information but also ensuring the meaning behind message is clearly transferred without ambiguity.

The efficiency and safety of any healthcare setting is closely linked to the efficacy of the internal communication (34). Failure in communication is considered an important factor in poor outcomes and near misses in healthcare setting. For instance, a communication breakdown was responsible for $24 \%$ of the errors in a study published by Rogers et al. (39). Communication failures are also frequently the cause of near misses (40).
Sometimes it is easier to identify the barriers to effective communication (34) and to assume that in absence of barriers, communication can happen effectively.

However, communication does not happen when:

(I) The channel of communication does not exist/work or is not used;

(II) The channel exists but the important information is not transmitted;

(III) The channel exists, the information is transmitted but is misinterpreted by the recipient.

\section{Improving communication}

It is possible to improve the quality of communication focusing on the culture of the workplace and on the specific skills of the individuals. The individual feedback provided by communication experts after observations and assessments at the work place or during simulation can also be useful (41).

In addition to the training of healthcare professionals, the use of a structured way of conveying information helps both the sender and the receiver in the communication process. As examples, the use of checklists, structured handovers and team briefings all help to improve the quality and reduce the loss of relevant information during the communication process. 


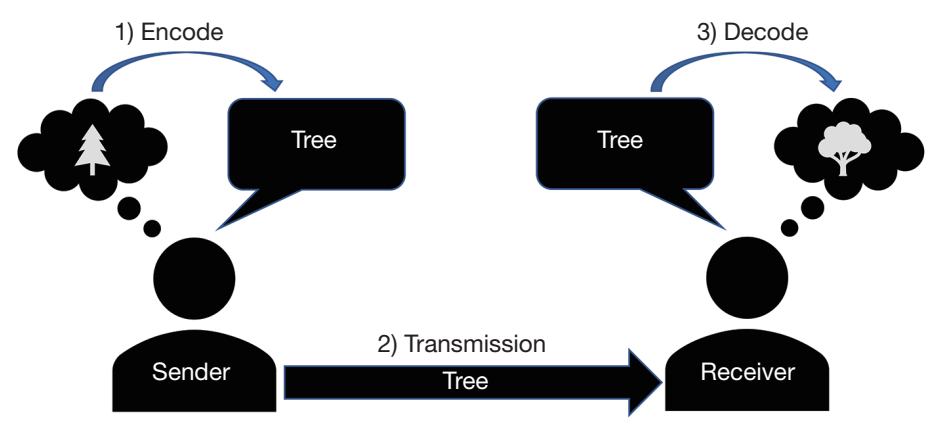

Model of one-way communication

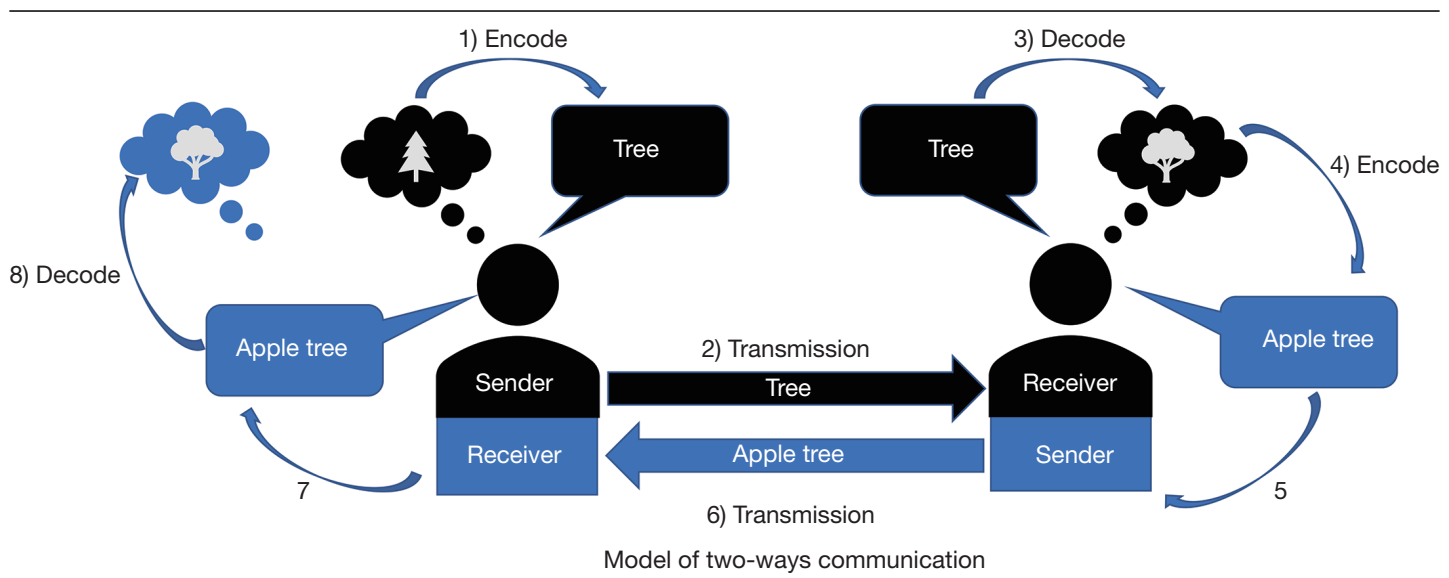

Figure 2 Simplified models of one-way and two-way communications.

Several communication models (Figure 2) have been identified based not-only on the use of verbal language and technical content but also on individual preferences toward certain types of information and non-verbal communication (42). Based on these elements, different strategies for team performance improvement have been developed (42).

\section{Teamworking}

Teams are important in healthcare because of the complicated and complex nature of diagnosis, treatment and recovery. It would be impossible to deliver the care needed without teams. However, teams are not simply a group of people working together, they are a group of two or more people that cooperate and collaborate in the pursuit of a common goal through effective communications, shared mental models, authentic leadership and a high level of trust.

The performance of a team does not just correlate with the technical skills of the individuals who are in the team, but also the effective application of non-technical skills which play an even more important role. Situation awareness of a team is not just the simple sum of the situation awareness of the individuals but the portion of the sum of awareness that is shared at every single moment in time. The decision-making process from the identification of the situation to the execution of the decision requires additional skills that include ability to lead, to coordinate, to follow...

The team we are part of are only a small number of the teams a patient sees during his/her treatment because, patient care in the $21^{\text {st }}$ century, is delivered by experts of multiple disciplines with different cultures. A thoracic patient, for example, will see during the perioperative period respiratory physicians, surgeons, oncologists, nurses (with different roles), physiotherapists, psychologists and many more. Consequently, there is a need to ensure that the information and knowledge pertaining to that patient is passed within and between the teams providing the care.

Elements that are important to the good functioning of a team includes the ability and willingness to support others, to solve conflict, to exchange relevant information and the coordination of activities. 


\section{How to teach, how to learn}

When introducing a new topic or concept into a domain, there is always a challenge in identifying what should be taught. Training needs analysis would normally be produced to determine the needs for each individual and team in the organization. However, when considering non-technical skills training for surgeons, there is already a significant body of evidence which describes the topics and how they should be taught so the wheel should not need reinventing. Whatever training program is delivered requires the framework of attitudes, knowledge and skills to be considered (43). The correct attitude towards the application and benefit of non-technical skills in the surgical environment is essential. This could be a significant challenge given previous experiences in adopting evidence-based programs (44). The next requirement is to build a solid foundation of knowledge before real practical skills development occurs.

A foundational package should develop a common vocabulary so that basic concepts are understood, and the culture of the 'tribe' is developed. This package can be delivered in a relatively didactic manner with student/staff engagement exploring knowledge boundaries using case studies and real-world examples. Knowledge reviews and assessments can provide a check of understanding.

There are two parts to the skills development process. The first part specifically focuses on non-technical skill development, but care must be taken to ensure that this learning is not 'contaminated' by focusing on technical skill development. Using training aids and specific nontechnical skills simulators such as GemaSim (45) have been shown as effective ways of facilitating this. The second part is to develop these non-technical skills in a relevant healthcare environment which allows representative challenges, pressures and drivers to be explored. Targeted objectives, potentially identified through competency-based assessments, could also be introduced at this stage. The relevance of this training to the surgical environment must be ensured through reference to case studies and faculty delivered and facilitated narratives.

The first courses written to specifically teach nontechnical skills were designed in aviation for pilots and crew members. For this reason, some healthcare courses use the acronym of CRM. In healthcare, the RCS (Ed) and RCA have developed and endorsed taxonomies and behavior rating system called NOTSS and anaesthetists nontechnical skills (ANTS) respectively to help develop skills in teams and individuals.
Based on the NOTSS and ANTS frameworks, the European Society of Thoracic Surgeons (ESTS) has identified a gap in the level of knowledge among European thoracic surgeons regarding non-technical skills. Therefore, ESTS has developed a course for surgeons that aims to provide a foundational level of knowledge concerning non-technical skills. The first course was run in 2017 and delivered with a series of monthly interactive webinars. The online environment offers the possibility to deliver information-based training and demonstration-based training. Ideally, once learned the basics of non-technical skills it would be ideal to move them into practice-based training that include simulation and role play.

\section{Conclusions}

A significant number of patients are injured every year while accessing medical and surgical care. This is similar to the situation of other high-risk industries at the end of the last century. The investigation of incidents in healthcare, as in other high-risk industries, has highlighted that, in many cases, they were not due to a lack of technical skills among professionals but to the complexity of the system and a number of failures in non-technical skills.

It is not easy to evaluate the results of teaching nontechnical skills per se, but the authors believe it is possible to measure the increase of safety in the system after its implementation. To start the change, several elements must be recognized as mandatory like the recognition of the limits of the humans involved in healthcare, the optimization of their non-technical skills and the design of cultural, organizational and physical environments that are safe for patients and healthcare professionals.

\section{Acknowledgments}

Funding: None.

\section{Footnote}

Provenance and Peer Review: This article was commissioned by the Guest Editor (Gilbert Massard) for the series "Training in Pulmonary Medicine and Surgery" published in Fournal of Thoracic Disease. The article has undergone external peer review.

Conflicts of Interest: All authors have completed the ICMJE uniform disclosure form (available at http://dx.doi. 
org/10.21037/jtd.2019.01.48). The series "Training in Pulmonary Medicine and Surgery" was commissioned by the editorial office without any funding or sponsorship. Dr. GC reports having no conflict of interest with the current paper. Nevertheless, he was working as a consultant surgeon for the National Health System in the UK when the paper was written and after he changed to Medical Doctor for Johnson and Johnson where he is currently working. The authors have no other conflicts of interest to declare.

Ethical Statement: The authors are accountable for all aspects of the work in ensuring that questions related to the accuracy or integrity of any part of the work are appropriately investigated and resolved.

Open Access Statement: This is an Open Access article distributed in accordance with the Creative Commons Attribution-NonCommercial-NoDerivs 4.0 International License (CC BY-NC-ND 4.0), which permits the noncommercial replication and distribution of the article with the strict proviso that no changes or edits are made and the original work is properly cited (including links to both the formal publication through the relevant DOI and the license). See: https://creativecommons.org/licenses/by-nc-nd/4.0/.

\section{References}

1. Leape LL, Brennan TA, Laird N, et al. The nature of adverse events in hospitalized patients. Results of the Harvard Medical Practice Study II. N Engl J Med 1991;324:377-84.

2. Mehtsun WT, Ibrahim AM, Diener-West M, et al. Surgical never events in the United States. Surgery 2013;153:465-72.

3. Massó Guijarro P, Aranaz Andres JM, Mira JJ, et al. Adverse events in hospitals: the patient's point of view. Qual Saf Health Care 2010;19:144-7.

4. Shorrock S. Never/zero thinking. 2016. Available online: https://humanisticsystems.com/2016/02/27/neverzerothinking/

5. Reason J. Human error: models and management. West J Med 2000;172:393-6.

6. Parker D, Wensing M, Esmail A, et al. Measurement tools and process indicators of patient safety culture in primary care. A mixed methods study by the LINNEAUS collaboration on patient safety in primary care. Eur J Gen Pract 2015;21 Suppl:26-30.

7. Smits M, Groenewegen PP, Timmermans DR, et al. The nature and causes of unintended events reported at ten emergency departments. BMC Emerg Med 2009;9:16.

8. Waring A. Safety Management Systems. London: Chapman \& Hall; 1996.

9. Haerkens MH, Kox M, Lemson J, et al. Crew Resource Management in the Intensive Care Unit: a prospective 3-year cohort study. Acta Anaesthesiol Scand 2015;59:1319-29.

10. Moffatt-Bruce SD, Hefner JL, Mekhjian H, et al. What Is the Return on Investment for Implementation of a Crew Resource Management Program at an Academic Medical Center? Am J Med Qual 2017;32:5-11.

11. Pittet D, Boyce JM. Hand hygiene and patient care: pursuing the Semmelweis legacy. The Lancet Infectious Diseases 2001;1:9-20.

12. Marshall MB, Emerson D. Patient safety in the surgical setting. Thorac Surg Clin 2012;22:545-50.

13. Kohn LT, Corrigan J, Donaldson MS. To err is human: building a safer health system. Washington DC: National Academy Press; 2000.

14. Clark SC, Dunning J, Alfieri OR, et al. EACTS guidelines for the use of patient safety checklists. Eur J Cardiothorac Surg 2012;41:993-1004.

15. Novoa NM. Patient safety in thoracic surgery and European Society of Thoracic Surgeons checklist. J Thorac Dis 2015;7:S145-51.

16. Reid J, Clarke J. Progressing safer surgery. J Perioper Pract 2009;19:336-41.

17. Catchpole K, Russ S. The problem with checklists. BMJ Qual Saf 2015;24:545-9.

18. Russell T. Safety and quality improvement in surgical practice. Ann Surg 2006;244:653-5.

19. Fabri PJ, Zayas-Castro JL. Human error, not communication and systems, underlies surgical complications. Surgery 2008;144:557-63; discussion 63-5.

20. Dekker SW, Breakey H. 'Just culture:' Improving safety by achieving substantive, procedural and restorative justice. Safety Science 2016;85:187-93.

21. Murdock D. Trauma: when there's no time to count. AORN J 2008;87:322-8.

22. Shappell S, Wiegmann D. The human Factors Analysis and Classification System - HFACS. Available online: https://commons.erau.edu/cgi/viewcontent.cgi? article=177 $7 \&$ context=publication

23. Olsen NS. Coding ATC incident data using HFACS: Inter-coder consensus. Safety Science 2011;49:1365-70.

24. Pupulidy I, Vesel C. The learning review: Adding to the accident investigation toolbox. Available online: http:// 
www.safetydifferently.com/wp-content/uploads/2018/08/1 71024TheLearningReview.pdf

25. Yule S, Paterson-Brown S. Surgeons' non-technical skills. Surg Clin North Am 2012;92:37-50.

26. Clinical Human Factors Group. Human Factors In Healthcare: Common Terms. 2016. Available online: https://drive.google.com/file/ d/0B4dbLgB56hptUGlLbHBOU3hkNmc/view

27. Flin R, Youngson G, Yule S, et al. Non technical skills for surgeons (NOTTS) System Handook v1.2. Royal College of Surgeons of Edinburgh and NHS Education for Scotland 2012. Available online: https://www.rcsed.ac.uk/ media/4605/notss-handbook-2012-no-bleeds.pdf

28. Graham J, Hocking G, Giles E. Anaesthesia nontechnical skills: Can anaesthetists be trained to reliably use this behavioural marker system in 1 day? Br J Anaesth 2010;104:440-5.

29. Salas E, King H, Rosen M. Improving teamwork and safety: toward a practical systems approach, a commentary on Deneckere et al. Soc Sci Med 2012;75:986-9.

30. Endsley MR. Toward a Theory of Situation Awareness in Dynamic Systems. Hum Factors 1995;37:32-64.

31. Wickens CD. Attentional Tunneling and Task Management. Available online: https://corescholar. libraries.wright.edu/cgi/viewcontent.cgi? article $=1130 \&$ co ntext=isap_2005

32. Bromiley M. Clinical Human Factors Group. 2016. Available online: https://chfg.org/whats-the-point-ofclinical-human-factors/

33. Hyman N. Nature, causes and consequences of unintended events in surgical units (Br J Surg 2010; 97: 1730-1740). Br J Surg 2010;97:1741.

34. Flin RH, O'Connor P, Crichton M. Safety at the sharp

Cite this article as: Casali G, Lock G, Novoa NM. Teaching non-technical skills: the patient centered approach. J Thorac Dis 2021;13(3):2044-2053. doi: 10.21037/jtd.2019.01.48 end: a guide to non-technical skills. Burlington: Ashgate Publishing Limited; 2008.

35. Klein G, Calderwood R, Clinton-Cirocco A. Rapid Decision Making on the Fire Ground: The Original Study Plus a Postscript. J Cogn Eng Decis Mak 2010;4:186-209.

36. Snowden DJ, Boone ME. A Leader's Framework for Decision Making. Harv Bus Rev 2007;85:68-76.

37. Snowden DJ. Cynefin Framework. 2010. Available online: http://cognitive-edge.com/videos/cynefin-frameworkintroduction/

38. Van Beurden EK, Kia AM, Zask A, et al. Making sense in a complex landscape: how the Cynefin Framework from Complex Adaptive Systems Theory can inform health promotion practice. Health Promot Int 2013;28:73-83.

39. Rogers SO Jr, Gawande AA, Kwaan M, et al. Analysis of surgical errors in closed malpractice claims at 4 liability insurers. Surgery 2006;140:25-33.

40. Tourgeman-Bashkin O, Shinar D, Zmora E. Causes of near misses in critical care of neonates and children. Acta Paediatr 2008;97:299-303.

41. Gjeraa K, Spanager L, Konge L, et al. Non-technical skills in minimally invasive surgery teams: a systematic review. Surg Endosc 2016;30:5185-99.

42. Weinert M, Mayer H, Zojer E. Geschulte Kommunikation als "Intervention". Der Anaesthesist 2015;64:137-44.

43. Baker DP, Amodeo AM, Krokos KJ, et al. Assessing teamwork attitudes in healthcare: development of the TeamSTEPPS teamwork attitudes questionnaire. Qual Saf Health Care 2010;19:e49.

44. Smith J. From optimism to hubris. BMJ 2004;329:0.

45. Kaiser D, Eberhart J, Butler C, et al. Real Time Cockpit Resource Management (CRM) Training. 2012. Available online: https://apps.dtic.mil/dtic/tr/fulltext/u2/a542117.pdf 